\title{
Blood Oxygenation Level-Dependent Functional MRI of Early Evidences of Brain Plasticity after Hemodialysis Session by Helixone Membrane of Patients with Indices of Adrenal Deficiency
}

\author{
Saïd Boujrafa, b Rachida Belaïch ${ }^{a, b}$ Abdelkhalek Housni ${ }^{b}$ \\ Mustapha Maaroufi ${ }^{b, c}$ Siham Tizniti ${ }^{b, c}$ Tarik Sqallid \\ Mohammed Benzagmout ${ }^{\mathrm{b}}$ \\ a Department of Biophysics and Clinical MRI Methods, and ${ }^{b}$ The Clinical Neuroscience Laboratory, Faculty of \\ Medicine of Fez, ${ }^{C}$ Department of Radiology and Clinical Imaging, and d Department of Nephrology, University \\ Hospital of Fez, Fez, Morocco
}

\section{Keywords}

Brain plasticity - Oxidative stress $\cdot$ Chronic renal failure - Hemodialysis - Adrenal gland deficiency . Blood oxygenation level dependent functional MRI

\begin{abstract}
Background: Various alterations of hypothalamic-pituitary-adrenal axis function have been described in patients with chronic renal failure. Nevertheless, controversial evidences were stated about the association between adrenal function deficiency (AD) and hemodialysis (HD). Purpose: The goal of this paper was to estimate indirect indices of the adrenal gland dysfunction which is potentially influenced by oxidative stress (OS) that still generates brain plasticity and reorganization of the functional control. Methods: Two male patients undergoing HD by the synthetic Helixone membrane for more than 6 months at the HD Center of the University Hospital of Fez, Fez, Morocco, were recruited. They underwent identical assessment immediately before and after the full HD session; this consisted of a blood ionogram revealing rates of sodium and calcium, and brain blood oxygenation level-dependent functional MRI (BOLD-fMRI) using
\end{abstract}

a motor paradigm in block design. Results: The blood ionogram revealed hypercalcemia and hyponatremia in both patients. Both biological assessment and BOLD-fMRI study results revealed a high level of OS that induced activation of a significantly large brain volume area suggesting the occurrence of possible brain plasticity and functional control reorganization induced by free radicals and enhanced by AD. Conclusion: The occurrence of brain plasticity and functional control reorganization was demonstrated in both patients studied who were undergoing HD by BOLD-fMRI with a notable sensitivity; this plasticity is induced by elevated OS occasioned by HD technique itself and probably amplified by AD. Similar results were found in a previous study performed on the same patients undergoing HD by a polysulfone membrane.

(c) 2017 S. Karger AG, Basel

\section{Introduction}

Various alterations of the hypothalamic-pituitary-adrenal axis function have been described in patients with chronic renal failure (CRF). Nevertheless, controversies

\section{KARGER}

(C) 2017 S. Karger AG, Basel

E-Mail karger@karger.com

www.karger.com/aon
Prof. Dr. Saïd Boujraf

Department of Biophysics and Clinical MRI Methods

Faculty of Medicine of Fez

BP 1893, Km 2.200, Sidi Hrazem Road, Fez 30000 (Morocco)

E-Mailsboujraf@gmail.com 
still remain regarding the occurrence of adrenal function deficiencies (ADs) in hemodialysis (HD) patients suffering of CRF [1-8].

Indeed, the $\mathrm{AD}$ is an uncommon challenging pathology particularly in patients suffering of CRF and undergoing $\mathrm{HD}$ [7-10], since the most clinical symptoms of $\mathrm{AD}$ are similar to those routinely found in patients suffering of AD like loss of weight, low blood pressure, anorexia, physical and psychological asthenia [7-9]. However, the most frequent biological disorders found in that patients include hyperkalemia, hyponatremia, and hypercalcemia [9].

Besides, the contact of blood with HD membrane causes several side effects in CRF patients, including the generation of oxidative stress (OS) with excessive production of free radicals [7-12]. Consequently, OS has a direct impact on the brain tissue since it is involved in most metabolic processes [7-19], as well as brain plasticity and reorganization of the functional control $[13,14$, $20,21]$. Additionally, the adrenal gland (AG) is potentially influenced by OS and other factors such neuropeptides and neurotransmitters [18].

The most used methods for assessing OS in patients suffering from CRF is the thiobarbituric acid assay that measures malondialdehyde acid, one of the major reactives resulting from the lipid peroxidation [19]. Besides, it was recently established that the blood oxygenation level-dependent functional MRI (BOLD-fMRI) was an efficient technique for evaluating OS in chronic HD patients $[13,14,20-25]$. Hence, the goal of this paper was to evaluate indirect indices of the AG dysfunction in patients undergoing $\mathrm{HD}$ by the synthetic helixone membrane. This $\mathrm{AD}$ is potentially influenced by OS, which generates brain plasticity and functional control reorganization.

\section{Methods}

\section{Patient's Characteristics}

Two patients were recruited in the Hemodialysis Center, University Hospital of Fez, Fez, Morocco. They gave consent to participate to our fMRI study conducted by Clinical Neuroscience Laboratory, Faculty of Medicine and Pharmacy of Fez, University of Fez in collaboration with Nephrology Department, Radiology and Clinical Imaging Department, and the Central Laboratory of the University Hospital of Fez, Fez, Morocco [7, 8, 13, 14, 20, 21].

Both patients were men who were undergoing chronic HD for more than 6 months. The duration of HD before recruitment was, respectively, 48 and 24 months. They were, respectively, 25 and 29 years old. Both patients underwent meticulous clinical and biological assessments; both did not show any signs of diabetes, tobacco use, infection episodes, or treatment with iron or erythropoietin injection.

BOLD-fMRI of Brain Plasticity after HD

Session by Helixone Membrane

\section{Biological Assessment}

Patients underwent identical assessment protocol immediately before starting HD session and immediately after achieving a full HD session. Blood ionogram assessing sodium $(\mathrm{Na})$ and calcium (Ca) was done using Olympus AU640/AU400 apparatus in the central laboratory of the University Hospital of Fez, Fez, Morocco.

The detailed results were reported in the medical doctoral thesis by Batta [21]. Nevertheless, we have not been able to assess the level of AG hormones in these patients; and inspection of $\mathrm{AD}$ was based only on blood ionogram and was revealed months after patient's recruitment.

\section{BOLD-fMRI Acquisition Protocol}

Patients underwent identical brain BOLD-fMRI protocol before starting HD and after achieving the same HD session in the Hemodialysis Center of the University Hospital of Fez, Morocco. The image data were acquired in MRI Unit, Radiology and Clinical Imaging Department of the University Hospital of Fez, Morocco.

Brain BOLD-fMRI and anatomical MRI data were acquired using a 1.5-Tesla MRI system (Sigma, General Electric; Milwaukee, WI, USA). The acquisition of images was done using single-shot gradient-echo echo-planer imaging sequence. This approach was shown to be very sensitive to $\mathrm{T} 2 *$ effect generated during BOLD effect reflecting functional activity of the cortical brain tissue [13, 14, 20-22]. BOLD-fMRI acquisition parameters were the following: echo time $\mathrm{TE}=55 \mathrm{~ms}$, repetition time $\mathrm{TR}=3,000 \mathrm{~ms}$, slice thickness $=5 \mathrm{~mm}$, field of view $F O V=240 \mathrm{~mm}$, and 31 axial slices were acquired covering the whole brain. The acquisition matrix size was $128 \times 128$.

Each brain volume was acquired within $3 \mathrm{~s}$ while during the whole BOLD-fMRI acquisition protocol, 60 brain volumes were acquired within $3 \mathrm{~min}$. BOLD-fMRI paradigm consists of a simple motor task, where patients are asked to perform finger taping of the right hand during a continuous scanning session, in blocks of $30 \mathrm{~s}$ ON alternating with $30 \mathrm{~s}$ OFF. Target forces and rates of finger taping were constant within each $30 \mathrm{~s}$ block, for 10 repetitions.

\section{Post-Processing Data and Imaging Result Generation}

Image processing and statistical analysis were conducted with Statistical Parametric Mapping package version 8 (SPM8, 2008; http://www.fil.ion.ucl.ac.uk/spm; Wellcome Department of Cognitive Neurology, London, UK). All fMRI data were analyzed using general linear model approach as in SPM, and all volumes obtained were used for data analysis.

The pre-processing with SPM8 included realignment, co-registration, and spatial normalization in the template of Montreal Neurological Institute (MNI).

The gradient echo-planar images were realigned using a rigid body transformation to the first volume of the time series for each subject. Afterwards, data were spatially smoothed with a Gaussian filter (FWHM $8 \times 8 \times 8 \mathrm{~mm}$ ) and spatially normalized.

The cerebral activation was rendered either onto T1-weighted brain slices or on the surface of a standard brain of MNI, Montreal, QC, Canada. The box-car designed task was used and convolved with the functional hemodynamic response.

T-statistics was calculated for each voxel element, and $p<0.01$ was considered to be a statistically significant threshold for significantly activated areas that were correlated for multiple comparisons. The maximal BOLD signal changes were calculated for each subject in the primary motor area M1 before and after HD sessions. Activation maps were calculated and overlaid on anatomical images. 


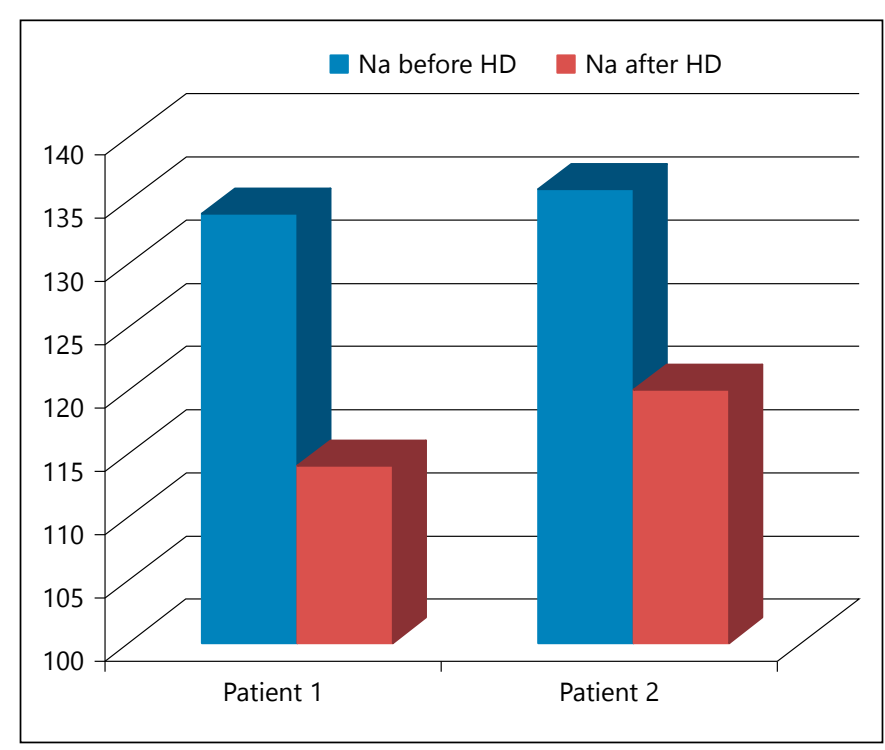

Fig. 1. Significant decrease of $\mathrm{Na}$ rate after $\mathrm{HD}$ session that well revealed hyponatremia.

\section{Results}

Presence of Indices of AG Dysfunction in Both Patients

The blood ionogram demonstrated a significant change in the rate of $\mathrm{Na}$ and $\mathrm{Ca}$ expressed as hypercalcemia and hyponatremia after HD sessions in both studied patients (Fig. 1, 2).

The Maximum Intensity of the BOLD Signal in the Activated Area Is Decreased after the HD Sessions

The maximum BOLD-fMRI signal in the individual activated brain areas of both patients has significantly decreased after HD sessions (Fig. 3); this decrease is visually well noticed in the 2-dimensional maps (Fig. 4). These maps represent typical functional BOLD-fMRI results.

These results was correlated to the OS while reflecting brain activation and functional control reorganization before and after HD session; this demonstrates a decrease in the localized hemodynamic response strength and expresses much localized decrease of metabolic involvement in brain functional activity of the primary motor area (M1). The same results were present in both patients. Finally, it is to consider that the maximum BOLD intensity is expressed in arbitrary unit.

\section{The Maximum Volume of BOLD Signal in the}

Activated Area Is Increased after the HD Sessions

A systematic and significant increase of the volume of the activated brain area involved in the motor task control

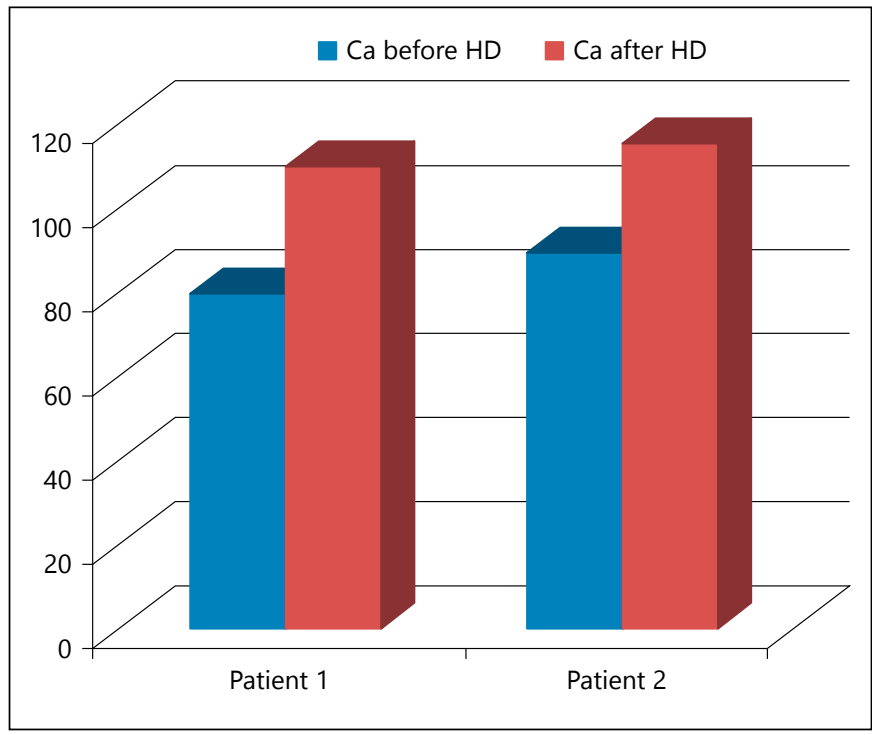

Fig. 2. Significant increase of Ca rate after HD session that revealed post dialysis hypercalcemia.

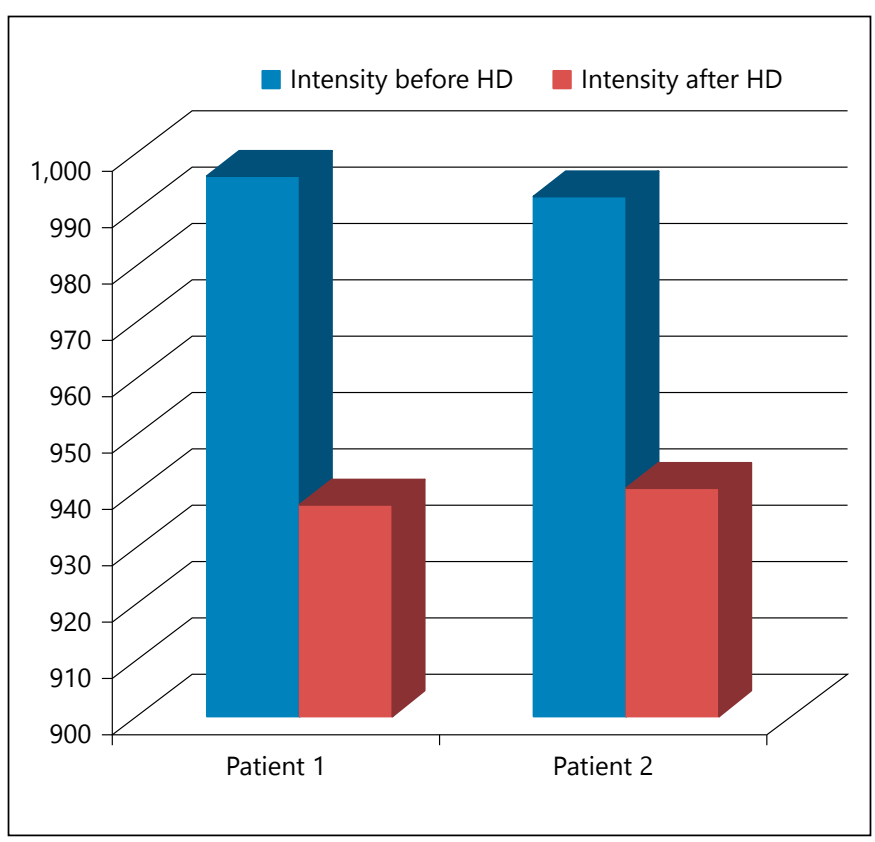

Fig. 3. A systematic decrease of the individual maximum localized BOLD signal in the primary motor area after HD session.

was noticed in both studied patients after HD sessions (Fig. 5).

Similarly, the typical 3D rendering results are well revealing important visual increase of the size of the volume brain activation (Fig. 6); this reflects a localized expanding hemodynamic response and an enlargement 


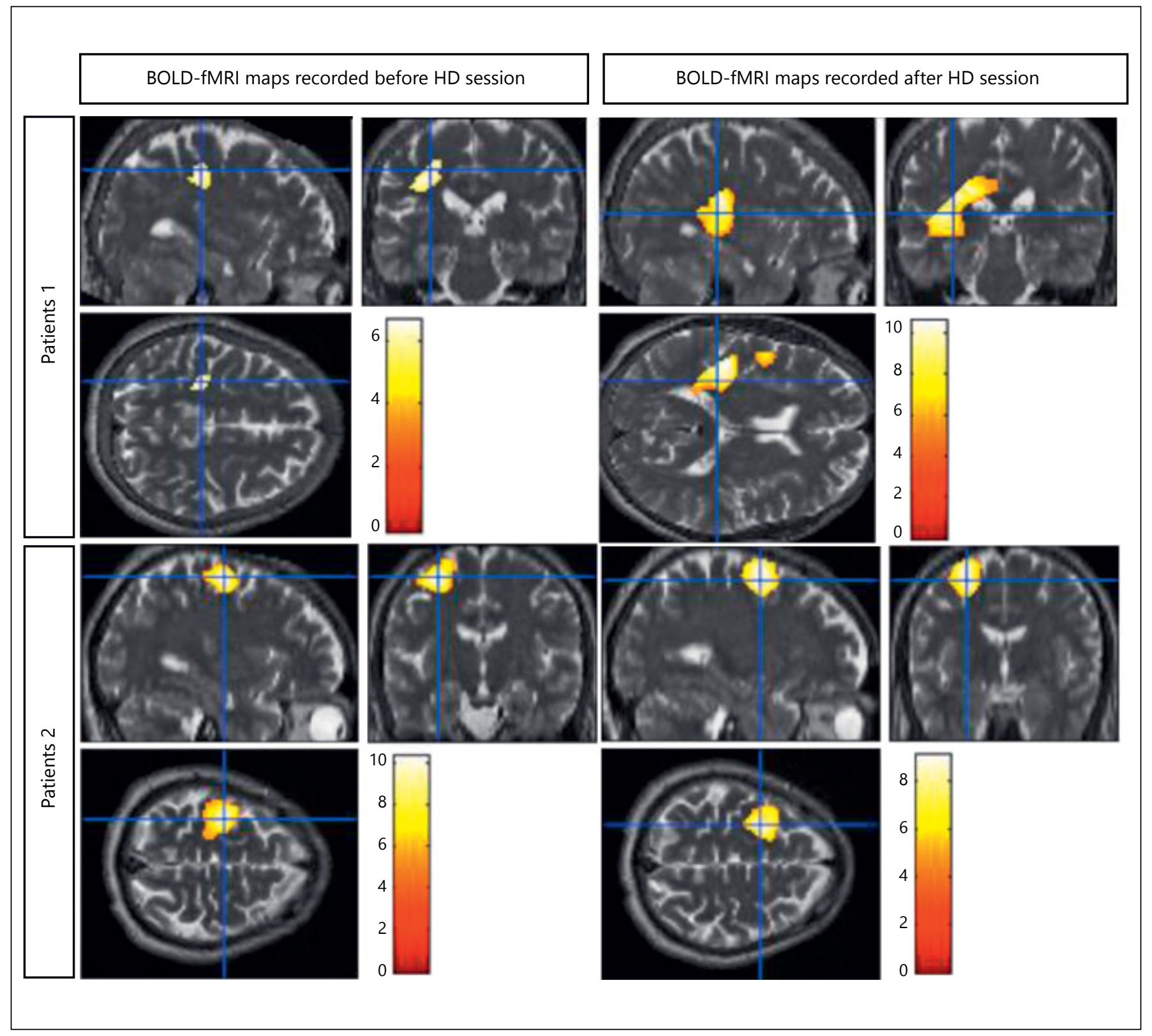

Fig. 4. Typical functional BOLD-fMRI $2 \mathrm{D}$ maps results are revealing visual decrease of the maximum BOLD-fMRI signal.

of localized increase of the metabolic activity, revealing thus an involvement of additional cortical brain tissue inside the primary motor area.

\section{Discussion}

The present study consisted of evaluating solely indirect indices of $\mathrm{AD}$ without assessing $\mathrm{AG}$ hormones in only 2 patients. Indeed, suspicion of $\mathrm{AD}$ was essentially based on blood ionogram results and has occurred months after patient's recruitment. However, these patients were lost on follow-up. Such critical statistical aspects would constitute a limitation of this study. However, the strength of this study consists of being, to the best of our knowledge, the first to demonstrate changes in brain function secondary to HD with helixone membrane in CRF patients with indirect indices of AD.

Indeed, OS is a state where the antioxidant systems are unable to correct spontaneously. Besides, oxygen sup- 


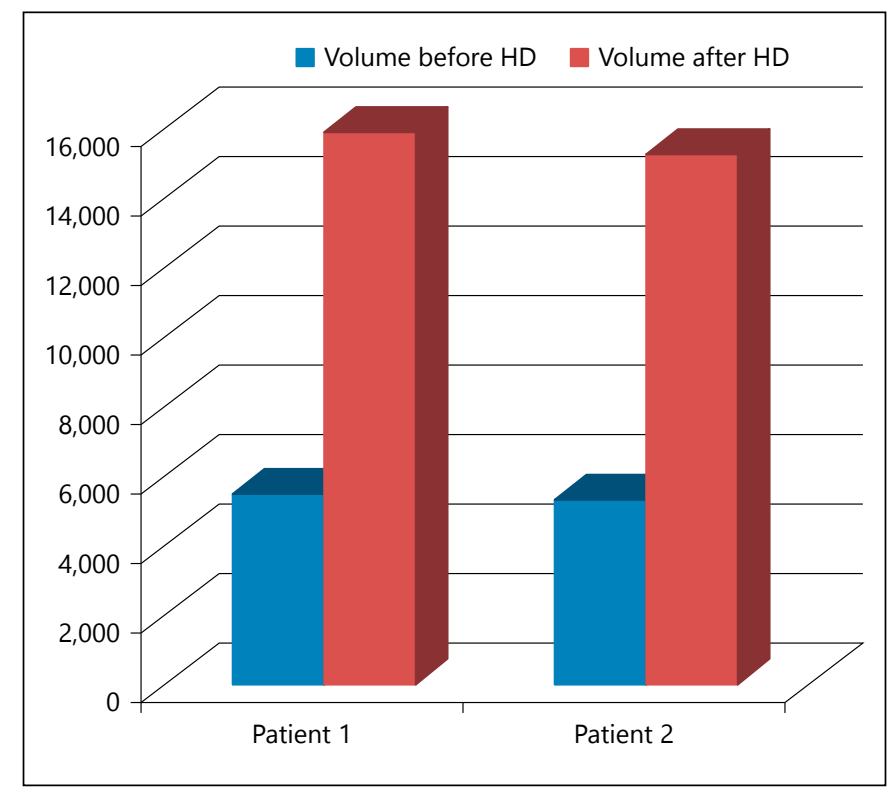

Fig. 5. A systematic increase of the localized BOLD-fMRI signal volume reflecting an increase of the volume of the activated brain area after HD session.

plied by the blood oxygenation process is behind OS phenomena whenever the control systems of metabolites generated are suffering or corrupted [25]. This imbalance leads to cell and tissue injury [12]. Since the brain is involved in most metabolic processes, it is highly exposed to OS $[13,14,20]$. Hence, BOLD-fMRI is a suitable imaging approach allowing spatial localization of the brain oxygenation change $[8-15,21]$. This technique is also used to assess BOLD phenomena that are directly linked to brain activity/plasticity and OS [7, 8, 13, 14, 20-29].

It is well-known that CRF disease itself is a direct factor originating OS before HD sessions $[7,8,13,14,20$ 24]. However, HD enhances the level of OS which is mostly amplified in brain tissue considering the higher oxidative metabolism engaged and lower antioxidant availability in the cerebral tissue $[24,30]$.

Numerous studies like our present study revealed that HD would lead to AD [11,31-33]. In fact, our patients might present an $\mathrm{AD}$ that should be confirmed by assessing the cortisol and the adrenocorticotrophic hormone rates. Nevertheless, this evaluation was not done in these patients and the diagnosis of adrenal insufficiency was based only on the blood ionogram results.

OS is basically generated by HD and amplified by both $\mathrm{HD}$ and $\mathrm{AD}[7,8,13,14,24-33]$. Besides, $\mathrm{HD}$ and $\mathrm{AD}$ have the potential to increase the oxidative damage to lipids, protein, and DNA, thus allowing the reduction of oxygen available within the blood network including brain microvasculature. Nevertheless, the exact mechanisms responsible for such effects are still not completely elucidated.

Basically, BOLD-fMRI contrast is based on magnetic susceptibility originated by the interaction of oxyhemoglobin and deoxyhemoglobin, which have very different levels of real time signal enhancement. Indeed, oxyhemoglobin is diamagnetic without any impact on the signal of MRI methods. Oxyhemoglobin is deoxygenated once the oxygen charge of the hemoglobin is consumed through local cellular and molecular metabolism processes. Deoxygenation of oxyhemoglobin generates deoxyhemoglobin that are molecular entities with unpaired electrons which has paramagnetic magnetic characteristics [13, 14, 20, 29, 34].

BOLD-fMRI measurement consists of reflecting the average transformation of oxyhemoglobin to deoxyhemoglobin in a given voxel of the brain, while this rate depends on the cerebral blood volume (CBV) and cerebral blood flow (CBF) $[13,14,20,29,34]$. Indeed, CBV and CBF are varying within brain tissues according to physiological conditions underlying a corresponding metabolism and molecular state $[13,14,20,29,34]$. Hence, increased OS might impair the transformation process of oxyhemoglobin to deoxyhemoglobin in given voxel of the brain $[13,14,20,29,34]$. Consequently, these transformation and conversion processes allow a significant and consistent variation of MRI signal [13, 14, 20, 29, 34].

Finally, measured BOLD-fMRI signal in different physiological, cellular, metabolic, and molecular conditions constitutes indirect measurement of the impact of such conditions in brain tissues $[13,14,20,29,34]$. Belaich and Boujraf [7] have suggested that fewer more radicals are produced in the mitochondria of cells because $\mathrm{HD}$ and suspected AD generally enhance energy utilization $[13,14,20,22]$. This last process is yielding conditions where tissues are exposed to more cellular oxidative damage [35].

In our study, a significant decrease in the intensity of brain activation of the primary motor area had occurred after HD; such brain activation was negatively correlated with levels of OS. This correlation was demonstrated by earlier studies that have assessed OS in CRF using BOLDfMRI but in renal tissue and not in the brain $[35,36]$.

Indeed, OS is predominantly originated by the blood contact with the HD membrane and the extracorporeal circuit $[7,8,12]$. This leads to multiple cellular alterations such as apoptosis and necrosis [12, 37]. Additionally, studies demonstrated the implication of OS in several neurodegenerative diseases, such as Alzheimer's disease and Parkinson's disease [38, 39].
Boujraf/Belaïch/Housni/Maaroufi/Tizniti/ Sqalli/Benzagmout 
Typical individual 3D rendering before HD session
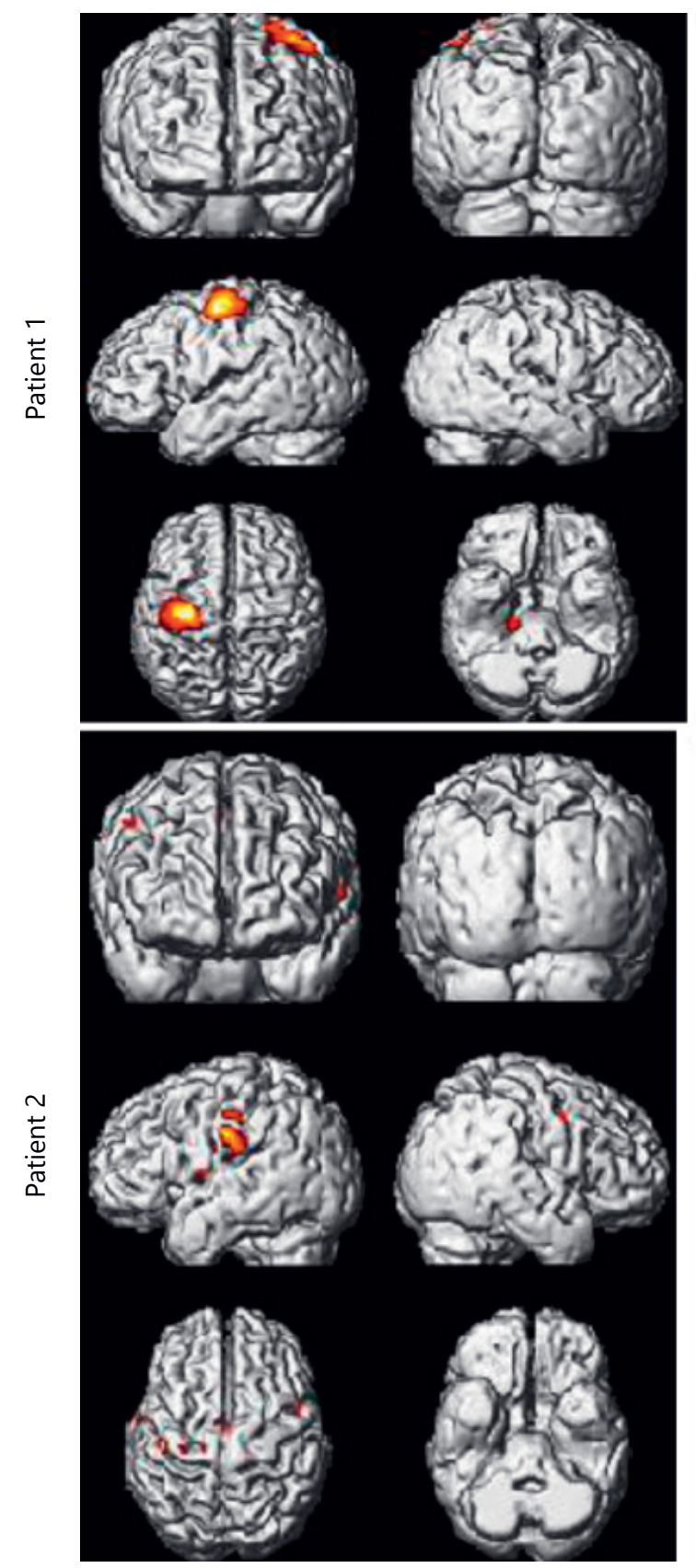

Typical individual 3D rendering after HD session
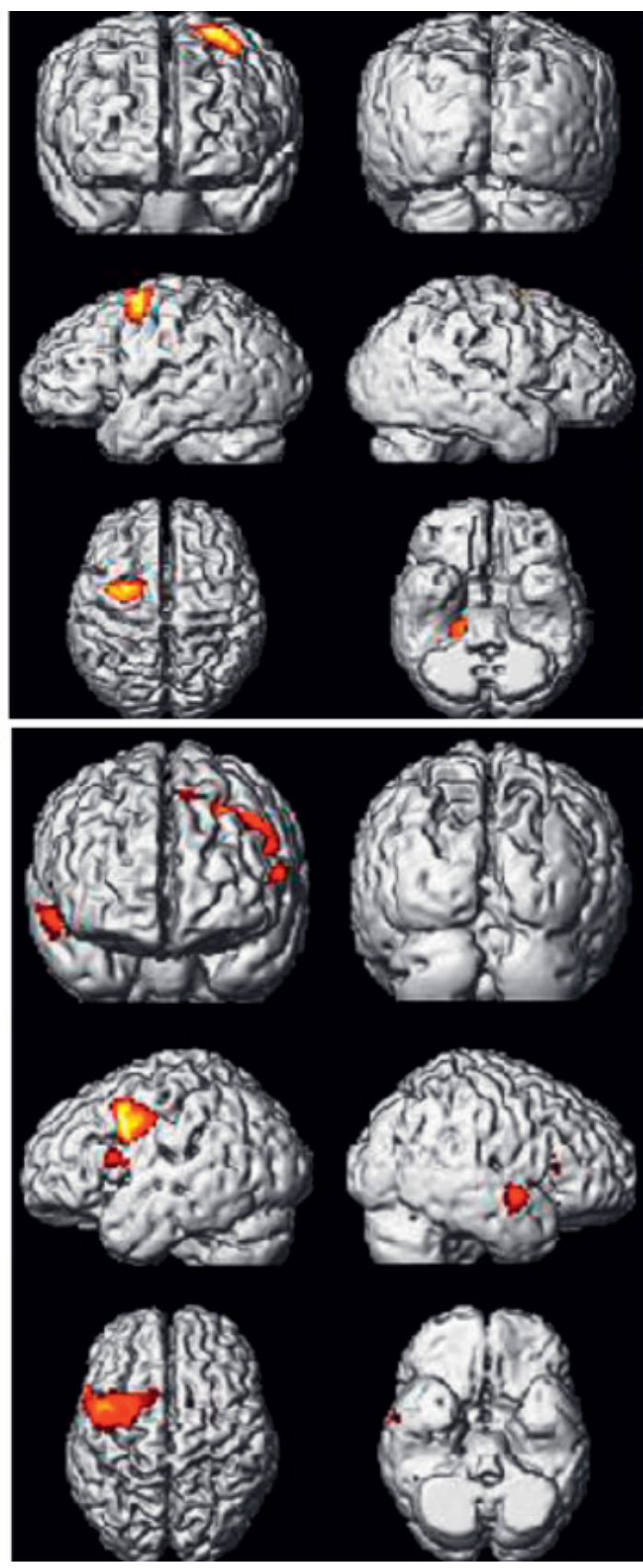

Fig. 6. Typical individual 3D rendering maps fitted on standard brain showing BOLD-fMRI activation of the motor cortex in each patient before and after HD sessions. 
Interestingly, our study showed that OS induced an activation of significantly larger volume area of brain suggesting thus the occurrence of brain plasticity and functional reorganization in these patients, and the phenomena was induced by free radicals. In fact, earlier studies have shown the implication of free radicals in the modulation of the synaptic plasticity [40,41], especially reactive oxygen species that might play the role of modulators and regulators of synaptic plasticity $[42,43]$.

Consequently, we can suggest that plastic brain changes were induced by $\mathrm{HD}$ process through modifications in the OS status and oxygen availability in localized tissue of the brain [43].

Consistently, both patients demonstrated brain plasticity and functional control reorganization after HD which was compared to similar patients subjects $[13,14$, $20,22]$. The unique particularity found in these patients was that the brain plasticity was amplified in patients with indices of AD. Finally, it should be emphasized that these results show indirectly that OS could be amplified by the presence of an $\mathrm{AD}$, leading to significant brain plastic changes revealed by BOLD-fMRI with a remarkable sensitivity.

\section{Conclusion}

Despite the very limited number of patients, this study is formally the first to demonstrate plastic changes in the brain that were originated by HD using helixone membrane in CRF patients with indirect indices of AD. Studied patients have had significant brain plasticity and functional control reorganization that was assessed by BOLD-fMRI with a remarkable sensitivity. These brain plastic changes were induced by higher OS state and amplified by AD.
Similar results were found in a previous study performed for the same patient population undergoing HD by polysulfone membrane. However, more evaluations and extensive studies should be achieved in similar conditions to precisely define the exact relationship between $\mathrm{HD}, \mathrm{AD}, \mathrm{OS}$, and brain plastic changes.

\section{Acknowledgments}

The authors acknowledge all the doctors, staff, and patients of the University Hospital of Fez, and Hemodialysis Center of Fez that have contributed directly or indirectly to this work.

This article complies with International Committee of Medical Journal editor's uniform requirements for manuscript.

\section{Authorship Contributions}

S.B.: study design, writing, study analysis, and reviewing; R.B.: first draft writing, study analysis; A.H.: technical support and supply; M.M.: patient's management and reviewing; S.T.: patient's management, technical and logistic support; T.S.: early study design and patients' recruitment and management; M.B.: data interpretation, data analysis, and critical reviewing.

\section{Disclosure Statement}

The initial BOLD-fMRI results were reported in the Master Thesis by R.B. under the supervision of S.B. The initial OS and clinical biology results reported in this paper were obtained within framework of MD thesis by F.B. that was officially supervised by S.B. F.B. is acknowledged in the Acknowledgment section.

The clinical biology assessment was done in the Central Laboratory of the University Hospital of Fez, Morocco; while the OS study was done in Pharmacology Department of the Faculty of Medicine and Pharmacy, University of Fez, Morocco. The Patient recruitment was supervised by the leader (T.S.) of the Nephrology Department, University Hospital of Fez, Morocco.

\section{References}

1 Ramirez G, Gomez-Sanchez C, et al: Evaluation of the hypothalamic hypophyseal adrenal axis in patients receiving long-term hemodialysis. Arch Intern Med 1982;142:1448-1452.

2 McDonald WJ, Golper TA, et al: Adrenocorticotropin-cortisol axis abnormalities in hemodialysis patients. J Clin Endocrinol Metab 1979;48:92-95.

3 Wallace EZ, Rosman P, et al: Pituitary-adrenocortical function in chronic renal failure: studies of episodic secretion of cortisol and dexamethasone suppressibility. J Clin Endocrinol Metab 1980;50:46-51.
4 Nolan GE, Smith JB, et al: Spurious overestimation of plasma cortisol in patients with chronic renal failure. J Clin Endocrinol Metab 1981;52:1242-1245.

5 Barbour GL, Sevier BR: Adrenal responsiveness in chronic hemodialysis. N Engl J Med 1974;290:1258.

6 Heaton A, Johnston DG, et al: Twenty-four hour hormonal and metabolic profiles in uraemic patients before and during treatment with continuous ambulatory peritoneal dialysis. Clin Sci (Lond) 1985;69:449457.
7 Belaich R, Boujraf S: Oxidative stress and inflammatory factors in hemodialysis: effects and strategies. Med Mal Metab 2016;10:3842.

8 Belaïch R, Boujraf S, et al: Impact of oxidative stress and inflammation in hemodialysis patients. Med Ther 2015;21:95-103.

9 Gmar-Bouraoui S, Achour A, et al: [Adrenocortical insufficiency in renal amyloidosis]. Ann Endocrinol (Paris) 2001;62(1 pt 1):4-6.

10 Malet-Pipo D, Otal P, et al: Etiologie chez l'adulte: insuffisances surrénaliennes. Med Ther Endocrinol Reprod 2000;2:408-416. 
11 Clodi M, Riedl M, et al: Adrenal function in patients with chronic renal failure. Am J Kidney Dis 1998;32:52-55.

12 Libetta C, Sepe V, et al: Oxidative stress and inflammation: implications in uremia and hemodialysis. Clin Biochem 2011;44:11891198.

13 Belaïch R, Boujraf S, et al: Indices of adrenal deficiency involved in brain plasticity and functional control reorganization in hemodialysis patients with polysulfone membrane: BOLD-fMRI study. J Integr Neurosci 2016; 15:191-203.

14 Belaïch R, Boujraf S, et al: Implications of oxidative stress in the brain plasticity originated by fasting: a BOLD-fMRI study. Nutr Neurosci 2016:1-8.

15 Hanafy KA, Selim MH: Antioxidant strategies in neurocriticalcare. Neurotherapeutics 2012; 9:44-55.

16 Noseworthy MD, Bray TM: Effect of oxidative stress on brain damage detected by MRI and in vivo 31P-NMR. Free Radic Biol Med 1998; 24:942-951.

17 Kishida KT, Klann E: Sources and targets of reactive oxygen species in synaptic plasticity and memory. Antioxid Redox Signal 2007;9: 233-244.

18 Bornstein SR: Predisposing factors for adrenal insufficiency. N Engl J Med 2009;360: 2328-2339.

19 Del Rio D, Stewart AJ, et al: A review of recent studies on malondialdehyde as toxic molecule and biological marker of oxidative stress. Nutr Metab Cardiovasc Dis 2005; 15:316328.

20 Belaïch R, Boujraf S, et al: Assessment of hemodialysis impact by polysulfone membrane on brain plasticity using BOLD-fMRI. Neuroscience 2015;288:94-104.

21 Batta FZ: Caractérisation de l'Impact de la BiocompatibilitédesMembranesd'Hémodialyseet leur Changement sur le Stress Oxydant: Etudes Biologique et par IRMf-BOLD. MD Thesis,
Faculty of Medicine and Pharmacy of Fez, Morocco, 2011, p 102.

22 Boujraf S, Benajiba N, et al: The impact of restricted diet on brain function using BOLDfMRI. Exp Brain Res 2006;173:318-321.

23 Descamps-Latscha B, Witko-Sarsat V: [Oxidative stress in chronic renal failure and hemodialysis]. Nephrologie 2003;24:377-379.

24 Christen T, Bolar DS, et al: Imaging brain oxygenation with MRI using blood oxygenation approaches: methods, validation, and clinical applications. AJNR Am J Neuroradiol 2013; 34:1113-1123.

25 Kohen R, Nyska A: Oxidation of biological systems: oxidative stress phenomena, antioxidants, redox reactions, and methods for their quantification. Toxicol Pathol 2002;30:620650.

26 Boujraf S, Summers P, et al: Ultrafast bold fMRI using single-shot spin-echo echo planar imaging. J Med Phys 2009;34:37-42.

27 Halder P, Brem S, et al: Electrophysiological and hemodynamic evidence for late maturation of hand power grip and force control under visual feedback. Hum Brain Mapp 2007; 28:69-84

28 Raichle ME, Snyder AZ: A default mode of brain function: a brief history of an evolving idea. Neuroimage 2007;37:1083-1090.

29 Sørensen A, Pedersen M, et al: BOLD MRI in sheep fetuses: a non-invasive method for measuring changes in tissue oxygenation. Ultrasound Obstet Gynecol 2009;34:687-692.

30 Massaad CA, Klann E: Reactive oxygen species in the regulation of synaptic plasticity and memory. Antioxid Redox Signal 2011;14: 2013-2054.

31 Luger A, Lang I, et al: Abnormalities in the hypothalamic-pituitary-adrenocortical axis in patients with chronic renal failure. Am J Kidney Dis 1987;9:51-54.

32 Ramirez G, Bittle PA, et al: The effects of corticotropin and growth hormone releasing hormones on their respective secretory axes in chronic hemodialysis patients before and after correction of anemia with recombinant human erythropoietin. J Clin Endocrinol Metab 1994;78:63-69.

33 Suzuki R, Morita H, et al: Adrenal insufficiency in a haemodialysis patient. NDT Plus 2010; 3:99-100.

34 Djamali A, Sadowski EA, et al: BOLD-MRI assessment of intrarenal oxygenation and oxidative stress in patients with chronic kidney allograft dysfunction. Am J Physiol Renal Physiol 2007;292:F513-F522.

35 Chandarana $\mathrm{H}$, Lee VS: Renal functional MRI: are we ready for clinical application? Am J Roentgenol 2009;192:1550-1557.

36 Thoeny HC, Kessler TM, et al: Renal oxygenation changes during acute unilateral ureteral obstruction: assessment with blood oxygen level-dependent $\mathrm{mr}$ imaging - initial experience. Radiology 2008;247:754-761.

37 Pupim LB, Himmelfarb J, et al: Influence of initiation of maintenance hemodialysis on biomarkers of inflammation and oxidative stress. Kidney Int 2004;65:2371-2379.

38 Von Arnim CA, Gola U, et al: More than the sum of its parts? Nutrition in Alzheimer's disease. Nutrition 2010;26:694-700.

39 Mandel S, Grünblatt E, et al: Neuroprotective strategies in Parkinson's disease: an update on progress. CNS Drugs 2003;17:729-762.

40 Radak Z, Kumagai S, et al: Effects of exercise on brain function: role of free radicals. Appl Physiol Nutr Metab 2007;32:942-946.

41 Betzen C, White R, et al: Oxidative stress upregulates the NMDA receptor on cerebrovascular endothelium. Free Radic Biol Med 2009; $47: 1212-1220$.

42 Hota SK, Hota KB, et al: Oxidative-stress-induced alterations in $\mathrm{Sp}$ factors mediate transcriptional regulation of the NR1 subunit in hippocampus during hypoxia. Free Radic Biol Med 2010;49:178-191

43 Habas C: [Physiological basis of functional MRI]. J Radiol 2002;83:1737-1741. 